\title{
CICLODIÁLISIS POSTRAUMÁTICA TRATADA CON LÁSER DIODO TRANSESCLERAL
}

\section{POST-TRAUMATIC CYCLODIALYSIS CLEFT TREATED WITH TRANSSCLERAL DIODE LASER}

\author{
CERIO-RAMSDEN CD ${ }^{1}$, MUÑOZ-NEGRETE FJ ${ }^{2}$, REBOLLEDA G ${ }^{2}$
}

\begin{abstract}
RESUMEN
Caso Clínico: Paciente varón de 28 años, que tras traumatismo contuso en su ojo izquierdo, presenta una hipotonía ocular persistente (presión ocular $4 \mathrm{mmHg}$ ) acompañada de pérdida de agudeza visual $(0,4)$, edema de papila y pliegues coriorretinianos a nivel macular. El grosor foveal, medido con tomografía de coherencia óptica, era de 326 micras. Mediante biomicroscopia ultrasónica se aprecia una desinserción de iris nasal y un desprendimiento coroideo de $360^{\circ}$. El cuadro no cede con tratamiento conservador, por lo que 10 meses tras el traumatismo se le realiza una ciclopexia transescleral con láser diodo de contacto. Un año tras la aplicación del láser, la agudeza visual era 1, la presión ocular $14 \mathrm{mmHg}$ y el grosor foveal 240 micras.

Discusión: La ciclopexia transescleral con láser diodo puede ser una alternativa eficaz para la resolución de ciclodiálisis postraumáticas que no responden a tratamiento médico.
\end{abstract}

Palabras clave: Ciclodiálisis, láser diodo transescleral, hipotonía ocular, maculopatía por hipotonía.

\begin{abstract}
Case report: A 28-year-old man who received a blunt trauma in his left eye, presented with persistent ocular hypotony (intraocular pressure: $4 \mathrm{mmHg}$ ) as well as loss of visual acuity (0.4), optic disc edema and chorioretinal folds in the macula. The foveal thickness, measured by optic coherence tomography, was 326 microns. Using ultrasound biomicroscopy, a desinsertion of the nasal iris and a $360^{\circ}$ choroidal detachment was observed. The hypotony did not respond to conservative treatment and 10 months after the trauma he underwent a transscleral cyclopexy with contact diode laser. One year after the laser treatment the visual acuity was 1.0 , the intraocular pressure $14 \mathrm{mmHg}$ and the foveal thickness 240 microns.

Discussion: Transscleral cyclopexy with diode laser may be an effective alternative treatment to resolve post-traumatic cyclodialysis that does not respond to medical treatment (Arch Soc Esp Oftalmol 2009; 84: 47-50).
\end{abstract}

Key words: Cyclodialysis, transscleral diode laser, ocular hypotony, hypotony maculopathy.

\footnotetext{
Recibido: 23/11/07. Aceptado: 9/1/09.

${ }^{1}$ Licenciada en Medicina. Servicio de Oftalmología. Hospital Comarcal de Laredo (Cantabria).

2 Doctor en Medicina. Servicio de Oftalmología. Hospital Ramón y Cajal. Madrid. España.

Correspondencia:

Francisco J. Muñoz Negrete

Hospital Ramón y Cajal

Servicio de Oftalmología

Ctra. Colmenar Viejo, km 9,100

E-mail: franciscojmunoz@telefonica.net
} 


\section{INTRODUCCIÓN}

La ciclodiálisis se produce por la desinserción del cuerpo ciliar desde el espolón escleral. Puede estar causada por traumatismos contusos o de forma iatrogénica tras cirugía filtrante del glaucoma. Como consecuencia de la misma se produce una comunicación entre la cámara anterior y el espacio supracoroideo, lo que determina hipotonía ocular acompañada o no de desprendimiento coroideo y pliegues coriorretinianos que si afectan a la mácula pueden producir pérdida de agudeza visual (AV).

El tratamiento pretende normalizar la presión ocular (PIO), lo que suele conllevar una mejoría de AV. Cuando el tratamiento médico conservador fracasa, existen múltiples opciones la mayoría de ellas quirúrgicas. Presentamos un caso en el que la aplicación tardía de láser diodo transescleral logró revertir la hipotonía y mejorar la AV.

\section{CASO CLÍNICO}

Paciente varón de 28 años de edad que sufre un traumatismo contuso en su ojo izquierdo durante una agresión. En la exploración inicial de urgencia se aprecia en dicho ojo una importante reducción de AV debido a un hifema, la PIO era $8 \mathrm{mmHg}$ y en el fondo de ojo se apreciaba un edema de Berlin. El paciente es tratado con reposo relativo, cicloplejía, corticoides tópicos y sistémicos. Tras la reabsorción del hifema, la AV mejoró a 1, pero la PIO era de
$4 \mathrm{mmHg}$, por lo que se continúa tratamiento con restricción de esfuerzos físicos, atropina $1 \%$ y corticoides tópicos, a pesar de lo cual la hipotonía ocular persiste $(4-6 \mathrm{mmHg})$, la $\mathrm{AV}$ se reduce llegando a 0,4 y se van desarrollando pliegues retinocoroideos y edema de papila (fig. 1A). La supresión de los corticoides tópicos tampoco mejoró el cuadro clínico.

El estudio gonioscópico muestra un ángulo abierto grado IV sin otras alteraciones. Diez meses tras el traumatismo se le realiza una biomicroscopía ultrasónica (BMU) que objetiva un desgarro de la raíz de iris en la zona nasal con desprendimiento ciliocoroideo de $360^{\circ}$ (fig. 2A). La tomografía de coherencia óptica (OCT) muestra un engrosamiento foveal (326 micras).

Dada la ausencia de respuesta al tratamiento conservador se decide practicar una ciclopexia transescleral con láser diodo de contacto utilizando la sonda G del láser diodo Oculight SLx (Iris Medical Instruments, Mountain View, California), El procedimiento fue realizado bajo anestesia retrobulbar y se aplicaron 2 hileras de 10 impactos cada una (potencia 3 watios, tiempo de exposición 3 segundos) sobre el área de ciclodiálisis descrita con la BMU.

En las primeras 24 horas no se observaron cambios en la AV ni en la PIO, pero a partir de la semana se observó un ascenso gradual de la PIO, siendo al $6^{\circ}$ mes de $12 \mathrm{mmHg}$, lo que se acompañó de mejoría de AV y reducción progresiva del edema de papila, pliegues coriorretinianos (fig. 1B) y del grosor foveal (tabla I). Mediante BMU se comprobó el cierre de la ciclodiálisis mediante una sinequia
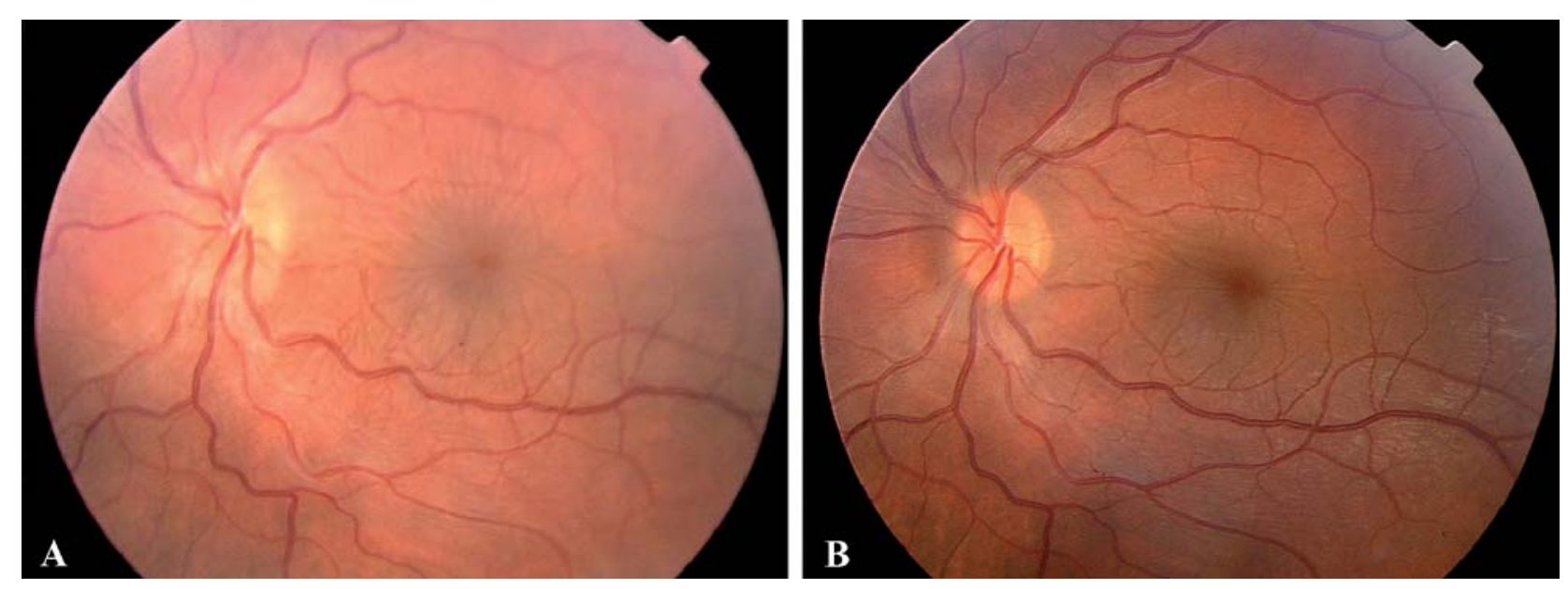

Fig. 1: A) Edema de papila y pliegues a nivel macular. B) Resolución del edema de papila y mejoría de los pliegues maculares 3 meses tras la aplicación del láser. 

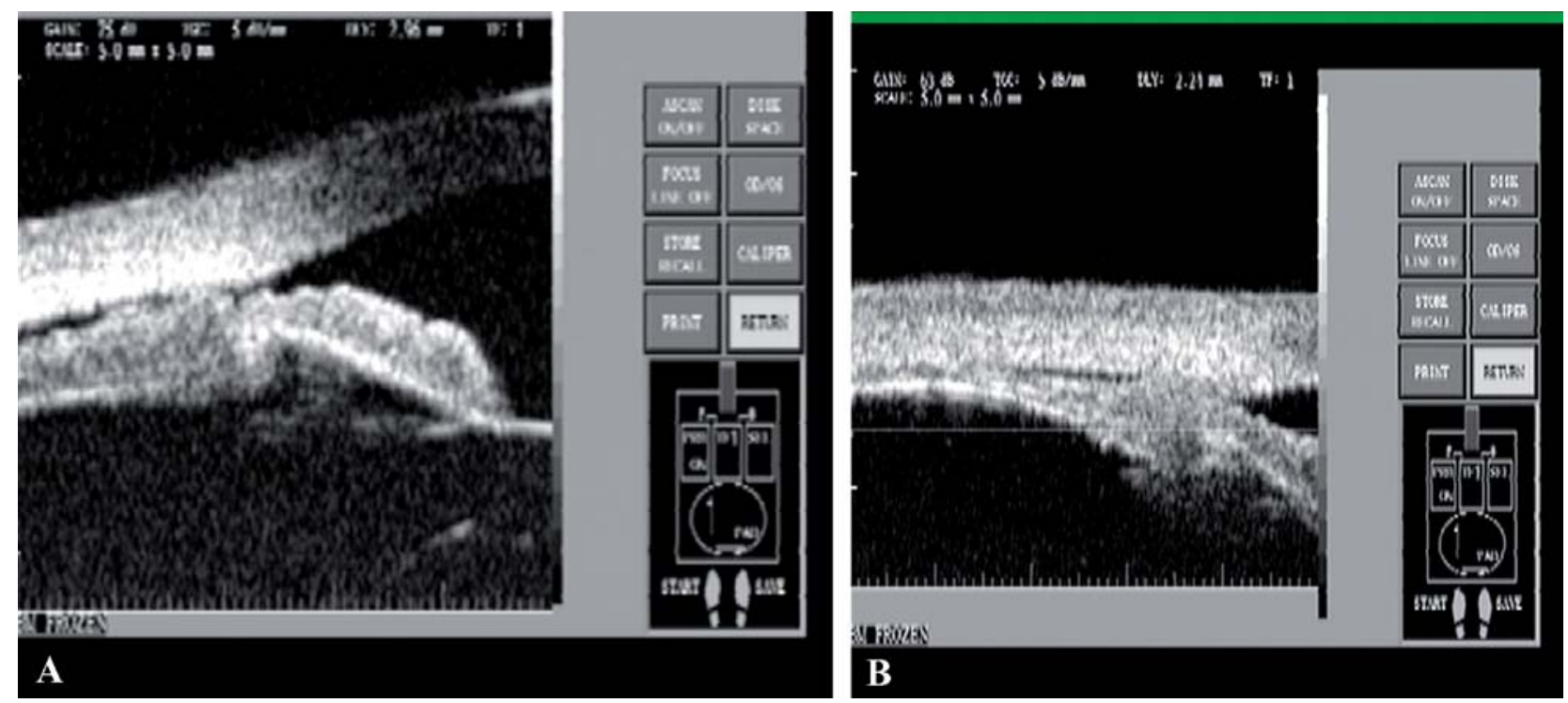

Fig. 2: A) Desgarro de la raíz del iris y desprendimiento coroideo. B) Sinequia anterior periférica y resolución del desprendimiento coroideo 6 meses tras la aplicación del láser.

anterior periférica y la desaparición del desprendimiento coroideo (fig. 2B), que justifican la normalización de la PIO. Al año de seguimiento la $\mathrm{AV}$ era de 1 y la PIO de $14 \mathrm{mmHg}$.

\section{DISCUSIÓN}

La ciclodiálisis establece una vía de salida directa para el humor acuoso al espacio supracoroideo, lo que determina una hipotonía ocular persistente, que puede comprometer la visión como consecuencia de complicaciones asociadas como desprendimiento coroideo, atalamia, pliegues coriorretinianos en región macular, edema del nervio óptico, éstasis venoso retiniano y catarata. La pérdida de visión secundaria a edema macular puede ser permanente si no es tratada a tiempo. Sin embargo, no está claro el tiempo a partir del cual se producen alteraciones irreversibles en la visión.

Dada su escasa prevalencia, es difícil establecer cuál es el tratamiento más adecuado de las ciclodiálisis. Todos los tratamientos pretenden recolocar la porción meridional del cuerpo ciliar sobre su inserción en el espolón escleral y así cerrar el flujo de humor acuoso hacia el espacio supracoroideo, causante de la maculopatía y edema de papila por hipotonía.

Inicialmente se recomienda tratamiento conservador con atropina $1 \%$ y dosis mínimas de corticoides. Aminlari et al recomiendan suspender el trata- miento con corticoides y proseguir con atropina $1 \%$ varias veces al día entre 6-8 semanas, en pauta descendente si mejora (1). En nuestro paciente tanto el tratamiento con corticoides tópicos como su supresión además del colirio de atropina no lograron resolver la ciclodiálisis. También se han descrito casos de curación espontánea (2). Un cierre espontáneo es raro que ocurra después de las 6 semanas. Aunque una demora de tratamiento mayor de 8 semanas podría aumentar el riesgo de pérdida de visión permanente, el tiempo que puede mantenerse no está claro y existen casos de recuperación de AV tras 7 años de maculopatía con hipotonía (3). En el presente caso a pesar de la demora de 10 meses en la resolución de la hipotonía logró normalizarse la AV.

Se han descrito múltiples tipos de cirugía: ciclopexia directa, diatermia, cerclaje escleral, vitrectomía crioterapia y taponamiento con gas, etc. No obstante, antes de recurrir a la cirugía se pueden utilizar

Tabla I. Evolución de diferentes parámetros tras la cicloplexia transescleral

\begin{tabular}{lccc}
\hline & PIO $(\mathrm{mm} \mathrm{Hg})$ & AV & Grosor foveal $(\mu)$ \\
\hline Pre & $4-6$ & 0,2 & 326 \\
$7 \mathrm{~d}$ & 12 & 0,4 & 314 \\
1 mes & 10 & 0,5 & 263 \\
3 mes & 11 & 0,6 & 240 \\
1 año & 14 & 1 & 240 \\
\hline \hline
\end{tabular}


tratamientos con láser. Joondeph (4) describe el uso de láser de argón en la zona de la diálisis, empleando 95-100 impactos de 100 micras de tamaño de estímulo, exposición de 0,1 segundos y 1-1,5 watios de potencia, que puede repetirse si es necesario. Para esta técnica se precisa una buena visualización gonioscópica del área a tratar. Suele acompañarse de picos hipertensivos dolorosos (1) que se resuelven con tratamiento médico. Otra modalidad es la endofotocoagulación con laser argón.

La ciclofotocoagulación con laser Nd:YAG transescleral (20 aplicaciones en 2 líneas de $6 \mathrm{~J}$ a 2$3 \mathrm{~mm}$ del limbo) y la crioterapia transconjuntival han sido utilizadas con éxito, pero también se acompañan de picos hipertensivos.

Amini (5) describe por vez primera el uso de ciclofotocaogulación transescleral con láser diodo en dos pacientes con ciclodiálisis persistente (un caso postraumático y otro tras cirugía filtrante con antimetabolitos) que se resolvieron tras la aplicación de 2 filas de 14 impactos (potencia de 2,5 w y tiempo de exposición de 2 segundos) sobre el área de la ciclodiálisis. En ninguno de los casos hubo pico hipertensivo en el postoperatorio. En nuestro caso aplicamos una mayor potencia (3 watios) y un tiempo de exposición más prolongado (3 segundos) para favorecer el efecto coagulativo del láser. El efecto del láser diodo sobre la ciclodiálisis puede observarse en la BMU en la que encontramos la aparición de una sinequia anterior periférica en la región afecta.

$\mathrm{Al}$ igual que en los casos previamente descritos, la utilización del láser diodo no se acompañó de pico hipertensivo postoperatorio, a diferencia del resto de procedimientos quirúrgicos para la ciclodiálisis. Una posible explicación puede residir en el hecho de que además de favorecer la adherencia del cuerpo ciliar se produzca una ablación parcial de los procesos ciliares que reduzca la producción de humor acuoso.
A pesar del tiempo prolongado transcurrido con hipotonía (10 meses), nuestro paciente presentó una progresiva mejoría de AV. Esta mejoría se correlacionó con una reducción del edema macular objetivado por OCT, por lo que hipotonías muy prolongadas pueden ser susceptibles de tratamiento y no producir efectos irreversibles para la visión.

La ciclopexia con láser diodo es una alternativa para el tratamiento de ciclodiálisis persistentes, que presenta enorme ventajas por ser un tratamiento sencillo, rápido y poco invasivo, que puede realizarse de forma ambulatoria. Al no requerir la entrada en globo ocular se evitan las infecciones postoperatorias. A diferencia de otros tratamientos no determina la producción de picos hipertensivos y tiene además la ventaja de no precisar visualización directa de la zona de ciclodiálisis, que puede localizarse previamente al tratamiento mediante BMU. En caso de fracaso, sería posible realizar un retratamiento o recurrir a otras alternativas terapéuticas más agresivas.

\section{BILBLIOGRAFÍA}

1. Aminlari A, Callahan CE. Medical, laser, and surgical management of inadvertent cyclodialysis cleft with hypotony. Arch Ophthalmol 2004; 122: 399-404.

2. González Martín-Moro J, Muñoz-Negrete FJ, Rebolleda $G$, Lara Medina J, García-Feijoó J. Hallazgos en biomicroscopía ultrasonica tras resolución espontánea de ciclodiálisis postraumática. Arch Soc Esp Oftalmol 2003; 78: 211-214.

3. Delgado MF, Daniels S, Pascal S, Dickens CJ. Hypotony maculopathy: improvement of visual acuity after 7 years. Am J Ophthalmol 2001; 132: 931-933.

4. Joondeph HC. Management of postoperative and posttraumatic cyclodialysis clefts with argon laser photocoagulation. Ophthalmol Surg 1980; 11: 186-188.

5. Amini H, Razeghinejad MR. Transscleral diode laser therapy for cyclodialysis cleft induced hypotony. Clin Experiment Ophthalmol 2005; 33: 348-350. 